\title{
Long-term reprogramming of the innate immune system
}

\author{
Jorge Dominguez-Andres $^{1} \quad$ Mihai G. Netea ${ }^{1,2,3}$
}

\author{
${ }^{1}$ Department of Internal Medicine and Radboud \\ Center for Infectious diseases (RCI), Radboud \\ University Nijmegen Medical Centre, Nijmegen, \\ The Netherlands \\ ${ }^{2}$ Department for Genomics \& Immunoregula- \\ tion, Life and Medical Sciences Institute (LIMES), \\ University of Bonn, Bonn, Germany \\ ${ }^{3}$ Human Genomics Laboratory, Craiova \\ University of Medicine and Pharmacy, Craiova, \\ Romania

\section{Correspondence:} \\ Jorge Dominguez-Andres, Department of Inter- \\ nal Medicine and Radboud Center for Infectious \\ diseases (RCI), Radboud University Nijmegen \\ Medical Centre, Geert Grooteplein 8,6500HB \\ Nijmegen, the Netherlands. \\ Email:jorge.dominguezandres@radboudumc.nl
}

\begin{abstract}
During the last few years, a growing body of evidence has shown that immunological memory is not an exclusive trait of lymphocytes, as many inflammatory insults can alter the functionality and the responsiveness of the innate immune system in the long term. Innate immune cells, such as monocytes, macrophages, dendritic cells, and NK cells can be influenced by the encounters with inflammatory stimuli, undergoing functional reprogramming and developing changed responses to subsequent chellenges. The long-term reprogramming depends on the rewiring of cell metabolism and epigenetic processes, and they stay at the basis of induction of both innate immune memory (also termed trained immunity) and innate immune tolerance. Here, we review the central role that the effects of this long-term reprogramming of innate immune cells plays in a number of clinically relevant conditions such as vaccination, atherosclerosis, sepsis, and cancer.
\end{abstract}

\section{KEYWORDS}

Innate immune system, trained immunity, metabolism, epigenetics, reprogramming, inflammation, vaccination, atherosclerosis, sepsis, cancer

\section{1 | INTRODUCTION}

The immune system is constantly challenged by external and internal stimuli since the day we are born. Every encounter between our immune cells and a stimulus leaves a footprint, shaping the originally naive immune system, which will progressively learn to differentiate between harmless stimuli and potentially pathogenic threats. Immunological memory has been clasically described for the adaptive immune system, in which naive B and T lymphocytes develop antigen-specific, long-lasting memory cells after encountering a new antigen. ${ }^{1}$ However, in the last years, a series of disoveries have demostrated that immunological memory is not an exclusive trait of lymphocytes. The function of cells from the innate immune system, such as monocytes, macrophages, dendritic cells (DCs), and NK cells, is also influenced by the contact with different stimuli, undergoing functional reprogramming, and facilitating a faster and enhanced response to future threats. ${ }^{2-4}$ Nevertheless, while the responses driven by $\mathrm{T}$ and B lymphocytes are antigen-specific, secondary responses involving innate

Abbreviations: Akt, protein kinase B; BCG, bacillus Calmette-Guérin; DCs, dendritic cells; DTP, vaccine against diphtheria, tetanus, and polio; EGFR, epidermal growth factor receptor; EgIN, hypoxia-inducible factor prolyl hydroxylase 2; H3K27ac, acetylation of histone 3 lysine 27; H3K4, histone 3 lysine 4; H3K4me3, trimethylation of histone 3 lysine 4; HIF1 $\alpha$, hypoxia-inducible factor $1 \alpha$; JmjC, jumonji C domain; MCV, measles-containing vaccines; mTOR, mammalian target of rapamycin; NLR, nucleotide-binding oligomerization domain-like receptors; OXPHOS, oxidative phosphorylation pathway; ROS, Reactive Oxygen Species; $\mathrm{SDH}$, succinate dehydrogenase; Srepb, Sterol regulatory element-binding transcription factor 1; TET, ten-eleven translocation enzymes cells are nonspecific and can be triggered by a wide variety of stimuli. The innate immunological memory in response to non-specific stimuli has beed termed systemic acquired resistance in plants, ${ }^{5}$ and trained immunity in vertebrates. ${ }^{6,7}$

Long-term shaping of the immune responses requires that the reprogramming induced by the first contact with the antigen is maintained long enough to enhance an active secondary response after a subsequent encounter with a pathogen. It has been widely described that memory lymphocytes can survive or self-renew for long periods of time. ${ }^{8,9}$ However, the mechanisms through which long-term innate immune memory is induced are still a matter of extensive research. Trained immunity involves profound reprogramming at metabolic, epigenetic, and transcriptional levels. ${ }^{10-12}$ In this review we summarize and discuss relevant contributions to the understanding of the processes involved in the functional reprogramming of the innate immune cells.

\section{2 | TRAINED IMMUNITY AND TOLERANCE}

A growing body of evidence has shown that the long-term epigenetic and metabolic reprogramming of innate immune cells plays a central role in a high number of clinically relevant conditions, such as vaccination, atherosclerosis, sepsis, or cancer. ${ }^{13-16}$ Anti-pathogenic responses of the innate cells are triggered after the involvement of pattern recognition receptors such as TLRs, nucleotide-binding oligomerization 


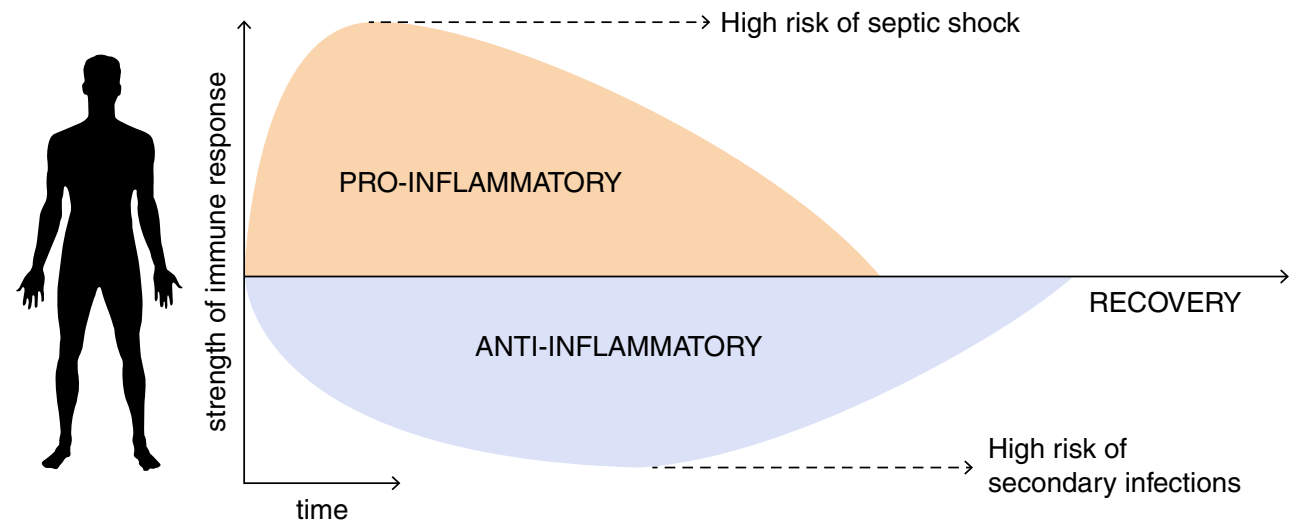

FIGURE 1 State of immune activation in patients with sepsis. The immune response during the acute phase of sepsis is characterized by exacerbated inflammation and the activation of immune effector mechanisms. Simultaneously, the immune system initiates anti-inflammatory mechanisms to reduce inflammation and promote tissue repair. The exaggerated systemic release of cytokines in the acute phase can lead to septic shock, with tissue damage, hypotension, cardiovascular dysfunction, and multiorgan failure. The disease activation of modulatory pathways may induce excessive tolerance and immunoparalysis, with the subsequent risk of secondary infections

domain-like receptors (NLR), or C-type lectins. ${ }^{17,18}$ However, these receptors also recognize nonpathogenic signals and induce sterile inflammation. ${ }^{19}$ In situations with excessive inflammation, tolerance acts as a mechanism that dampens the inflammatory response of the host to maintain homeostasis and prevent tissue damage and organ failure. ${ }^{20-22}$ Nevertheless, long lasting inhibitory effects in the immune function can also lead to a state of immunosuppression associated with a higher risk of secondary infections and a poorer outcome. ${ }^{23}$ Trained immunity and tolerance are the scales of a balance in which pro- and anti-inflammatory responses must be compensated to avoid either chronic inflammation or immune paralysis ${ }^{2}$ (Fig. 1). Tolerance also plays a crucial role in the regulation of physiological processes, as our immune system should not react against harmless stimuli, such as food antigens, inhaled allergens, or the gut microbiota. ${ }^{24}$

Trained immunity can be considered the opposite of the immune tolerance. Trained immunity involves an increase of the metabolic and epigenetic activity of the cell leading to an enhanced responsiveness to secondary stimulation. ${ }^{6}$ Tolerance is accompanied by a downregulation of the overall metabolic activities of the cell and a lack of accumulation of active histone marks at promoters and enhancers of genes related with immune functions. ${ }^{20}$ Subsequently, overly strong or longlasting activation of tolerance mechanisms can subsequently lead to a de facto immune paralysis with deleterious effects due to an increased susceptibility to secondary infections. ${ }^{24}$

Since the cells of the innate immune system have a short lifespan in circulation, ${ }^{25}$ there was an open question regarding how reprogramming of cells from this system could exert such long-lasting effects in the organism. Recent studies have shown that some stimuli induce systemic effects that have consequences several months after the first contact by altering hematopoiesis and triggering long-term reprogramming of progenitors in the bone marrow. ${ }^{26-28}$ Mitroulis et al. showed that $\beta$-glucan, the prototypical trained immunityinducing agonist, ${ }^{29}$ modulates hematopoietic stem and progenitor cells, influencing the behavior and responsiveness of peripheral myeloid cells, ${ }^{28}$ and similar observation was made by Kaufmann et al. after bacillus Calmette-Guérin (BCG) vaccination. ${ }^{27}$ Western-type diet-induced systemic inflammation led to prolonged enhancement of hematopoiesis and granulocyte-monocyte progenitor cell reprogramming in an IL-1 pathway-dependent fashion in an atherosclerosis mouse model. ${ }^{26}$ The long-term reprogramming of the cells of the innate immune system takes place at different levels, namely cell metabolism and epigenetic markers.

\section{3 | METABOLIC REPROGRAMMING IN INNATE IMMUNE CELLS}

The basal metabolism of innate immune cells greatly differs in function of the subset studied (Fig. 2). Overall, quiescent cells have low biosynthetic demands, and metabolic pathways are skewed toward metabolizing glucose through glycolysis coupled to oxidative phosphorylation. ${ }^{30}$ Upon activation, there is an increase in glucose uptake and glucose transformation into lactate through aerobic glycolysis, providing the cells with the precursors essential for the synthesis of nucleotides, amino acids, and lipids. ${ }^{31}$ Resting circulating monocytes mainly rely on the TCA cycle, whose products are employed as biosynthetic products for different molecules or as fuel for oxidative phosphorylation. ${ }^{32}$ Induction of trained immunity by fungal components such as $\beta$-glucan leads to a shift of cellular metabolism from oxidative phosphorylation toward aerobic glycolysis. ${ }^{10,11}$ This is a process mediated through the protein kinase $B(A k t) / m a m m a l i a n$ target of rapamycin (mTOR)/hypoxia-inducible factor $1 \alpha$ (HIF1 $\alpha$ ) pathway, which is crucial for an effective induction of trained immunity. ${ }^{10,11}$ This increased activity of the cell leads to an enhanced metabolic flux toward oxidative phosphorylation pathway (OXPHOS), where electrons transported by molecules, such as NAD+ and FADH, generate a gradient that will be used to synthesize ATP. ${ }^{33}$ In addition, the enhancement of the metabolic activity leads to the generation of considerable quantities of metabolites with immunomodulatory functions, such as fumarate, ${ }^{12}$ succinate, ${ }^{34}$ or itaconate, ${ }^{35,36}$ that also play a central role in the induction of long-term reprogramming of the cells of the innate immune system. 


\section{Basal metabolism (quiescent)}

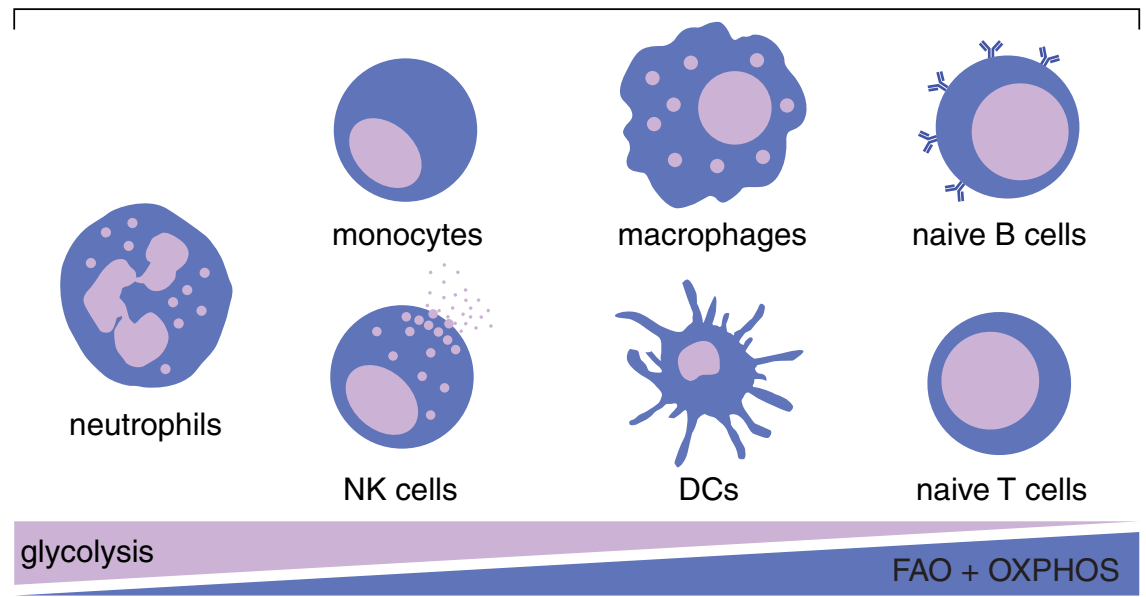

FIGURE 2 Classification of leukocytes in function of their basal metabolism in the quiescent state. Cells in the steady state have generally low biosynthetic demands and their basal metabolism is skewed toward metabolizing glucose through glycolysis coupled to oxidative phosphorylation. In this figure, cells are sorted from left to right, from more glycolysis-based metabolism to more OXPHOS and fatty acid oxidation-based basal metabolism. Left to right, resting neutrophils; resting myeloid cells (monocytes, macrophages, and dendritic cells) and NK cells; and naïve lymphocytes ( $T$ and $B$ cells)
The process of monocyte differentiation into macrophages is also associated with rewiring of cellular metabolism. Activated proinflammatory macrophages consume large quantities of glucose in order to fuel glycolysis and OXPHOS, both necessary to produce great amounts of proinflammatory cytokines and reactive oxygen species (ROS). ${ }^{37}$ Conversely, the metabolism of antiinflammatory macrophages is mainly mitochondrial and characterized by a blockade of glycolysis. ${ }^{31}$ In turn, neutrophils are highly reactive, short-lived cells, in which an active glycolytic metabolism allows to quickly induce oxidative burst, ROS, and the extrusion of neutrophil extracellular traps, while the functionality of their mitocondria are mainly oriented to maintain membrane potential through electron fluxes. ${ }^{38,39}$ The metabolism of dendritic cells changes during the different stages of development or activation. Development of DCs from precursors is associated with mitocondrial biogenesis, mainly driven by peroxisome proliferator-activated receptor $\gamma$, co-activator $1 \alpha$ (PGC1 $\alpha$ ), and mTOR. ${ }^{40,41}$ The basal metabolism of immature DCs relies on OXPHOS and fatty acid synthesis, ${ }^{42}$ while fully mature, activated DCs become highly glycolytic. ${ }^{43}$

In addition to myeloid cells, NK cells are lymphoid cells with innate caharcteristics that are very important for host defense, especially against virus infections. NK cells experience metabolic shifts upon activation in a stimulus-specific way. The rapid production of IFN- $\gamma$ in response to cytokines in an early activation phase requires functional oxidative phosphorylation while long-term adaptation relies both in enhanced glycolysis and oxidative phosphorylation. ${ }^{44,45}$ Granzyme $B$ production or degranulation were independent of metabolic changes. ${ }^{46}$ A recent study also showed that activated NK cells require the action of Srepb transcription factors to promote a metabolic pathway configuration in which glucose was metabolized to cytosolic citrate via the citrate-malate shuttle. ${ }^{47}$

The majority of studies on innate cell metabolism are focused on glycolysis and oxidative phosphorylation (Fig. 3). Beyond those, a number of recently released reports have shown that glucose-independent pathways also play a central role in cellular reprogramming after cell activation. Analysis of transcriptional data from macrophages stimulated with $\beta$-glucan revealed that the cholesterol synthesis pathway is highly up-regulated in trained immunity. ${ }^{10} \mathrm{~A}$ follow-up of this study showed that the activation of the cholesterol synthesis pathway, but not its synthesis itself, is crucial for innate memory and that statins prevent the induction of trained immunity by blocking mevalonate production. ${ }^{14}$ In agreement with this, the inhibition of cholesterol synthesis in mice reduced the induction of trained immunity by $\beta$-glucan. ${ }^{48}$ Fatty acids are the main constituents of triglycerides and phospholipids, which form cell membranes. The synthesis of fatty acids is closely related with glucose metabolism as it employs products of glycolysis, TCA cycle, OXPHOS, and the pentose phosphate pathway. ${ }^{49}$ Fatty acids can induce cellullar stress and activate innate immune pathways. Bone marrow-derived DCs and memory NK cells are characterized by fueling OXPHOS due to fatty acid oxidation. ${ }^{42,50}$ In LPS stimulated monocytes, the increase in NAD+/NADH ratio activates sirtuin 1 and 6, coordinating a switch to an anti-inflammatory state with increased fatty acid oxidation. ${ }^{51}$ Amino acids are the basic chemical building blocks during biogenesis, so they are of crucial importance in immune activity requiring cell division, cytokine, and chemokine production, but they can also act as precursors of different metabolites. Glutamine can be transformed into glutamate, and be subsequently transformed into $\alpha$-ketoglutarate, that can act as a source of succinate, fumarate, and citrate, which can replenish the TCA cycle. ${ }^{52}$ Glutamine replenishment of the TCA cycle leads to fumarate accummulation, inducing monocyte metabolic and epigenetic reprogramming by inhibiting KDM5 histone demehylases leading to enhanced secondary responses. ${ }^{12}$

\section{4 | EPIGENETIC REPROGRAMMING}

Together with the metabolic changes, stimuli-induced epigenetic reprogramming is the cornerstone of the induction and regulation of the innate immunological memory. Various epigenetic modifications are closely integrated with metabolic processes, as histone-modifying enzymes require metabolites as substrates or cofactors. DNA/histone methyltransferases require S-adenosyl methionine for their proper functioning; demethylases and ten-eleven translocation enzyme (TET) proteins are $\alpha$-ketoglutarate-dependent dioxygenases. ${ }^{53,54}$ HDACs and sirtuin SIRTs are NAD-dependent enzymes. ${ }^{55}$ Acetyl-CoA is the 


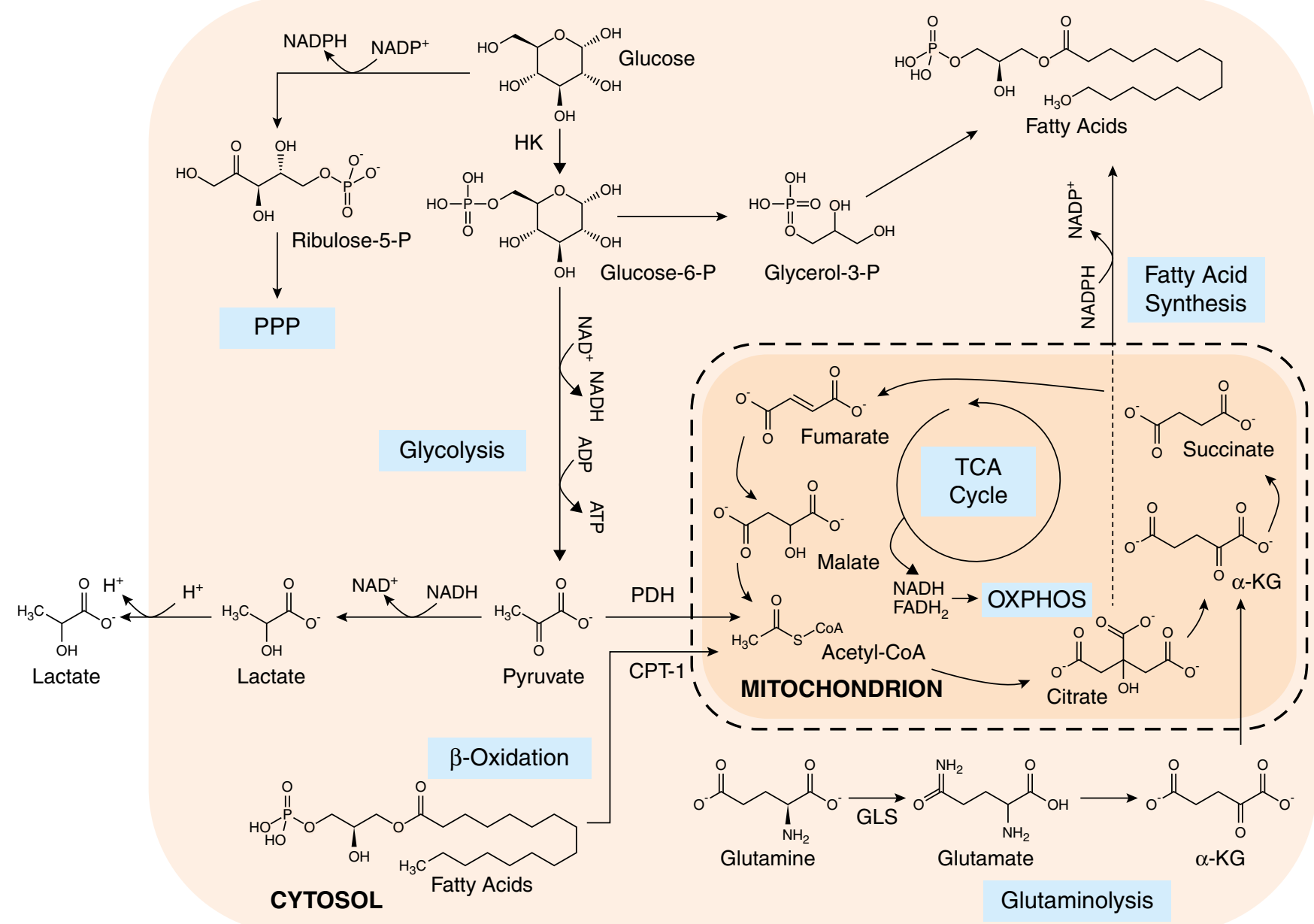

FIGURE 3 Overview of the principal intracellular metabolic pathways in innate immune cell metabolism and the main enzymes involved. Adapted from ${ }^{119}$

substrate used by histone acetyl transferase. ${ }^{56,57} \beta$-hydroxybutyrate is able to induce enrichment of histone acetylation and sustain a protective epigenetic state by inhibiting the activities of HDACs. ${ }^{58}$ Other $\alpha$-ketoglutarate-dependent dioxygenases, such as the EgIN prolyl hydroxylases, mark HIF transcription factor for destruction. ${ }^{59}$ HIF1a synthesis is controlled by the PI3K-AKT-mTOR axis, which senses nutrients such as glucose and amino acids, while its degradation is under control of EgIN prolyl hydroxylases, which respond to changes in oxygen and TCA cycle intermediates. Inhibition of succinate dehydrogenase (SDH) dysregulates histone modification in mammalian cells. ${ }^{60}$ Hydroxyglutarate, succinate, and fumarate are able to inhibit $\alpha$-ketoglutarate dependent dioxygenases, including the jumonji C domain histone demethylases and TET proteins. ${ }^{61-64}$

Terminally differentiated cells, such as monocytes and macrophages, modify their histone acetylation and methylation marks upon pathogen exposure, affecting their gene expression patterns upon subsequent stimulation. ${ }^{10,11}$ LPS induces histone modifications that lead to repressed proinflammatory gene expression after secondary stimulation, a feature of immunological tolerance. ${ }^{65}$ Many of the pathogen-induced epigenetic changes in macrophages persist after withdrawal of the stimulus and the removal of the transcription factors responsable for the initial deposition, namely mono-methylation of lysine(K)-4 on histone $3 .{ }^{66}$ Candida albicans- and $\beta$-glucan-induced functional reprogramming induced stable changes in histone trimethylation at $\mathrm{H} 3 \mathrm{~K} 4$ in human cells, showing functional relevance in a mouse in vivo model of infection. ${ }^{6}$ A follow-up of this study showed that $\beta$-glucan-induced changes in trimethylation of histone 3 lysine 4 (H3K4me3) and acetylation of histone 3 lysine 27 (H3K27ac) in human monocytes 7 days after the first stimulation in vitro were associated with a switch to glycolysis, suggesting a deep, long lasting reprogramming of the cells. ${ }^{11}$ Saeed et al. showed a strong correlation between $\mathrm{H} 3 \mathrm{~K} 27 \mathrm{ac}$ and $\mathrm{H} 3 \mathrm{~K} 4 \mathrm{me} 3$ changes during trained immunity, being $\mathrm{H} 3 \mathrm{~K} 27 \mathrm{ac}$ the most dynamic mark in human cells in vitro. ${ }^{10}$ Among the genes displaying increased $\mathrm{H} 3 \mathrm{~K} 27 \mathrm{ac}$, there was an enrichment in genes encoding for proteins involved in cellular signaling and inflammation: cytokines and chemokines, epidermal growth factor receptor and vascular endothelial growth factor pathways. The PI3K/AKT pathway was also shown to be up-regulated, fact supported by studies demonstrating the central role of this pathway in the induction of trained immunity by both BCG and $\beta$-glucan. ${ }^{11,48}$ Arts et al. showed in human monocytes in vitro and ex vivo that the epigenetic changes observed in BCG-trained monocytes are dependent on the induction of the metabolic pathways: if glycolysis or glutaminolysis is inhibited, changes in $\mathrm{H} 3 \mathrm{~K} 4 \mathrm{me} 3$ and $\mathrm{H} 3 \mathrm{~K} 9 \mathrm{me} 3$ at 
promoter sites of IL6 and TNFA are reversed, showing a link between these two regulatory cellular processes. ${ }^{12,48}$ The epigenetic profile induced by different stimuli shows close similarities among them, suggesting that there is a core trained immunity response induced by different stimuli.

\section{5 | CLINICALLY RELEVANT CONDITIONS OF LONG-TERM INNATE IMMUNE REPROGRAMMING}

\section{1 | Vaccination}

The WHO defines a vaccine as a biological preparation that improves immunity to a particular disease. In line with this definition, the concept of vaccination has clasically been linked to specificity, and therefore, to adaptive immunity. Nevertheless, the beneficial effects of multiple vaccines go far beyond the specific effects against the targeted diseases, but they can also increase resistance to other pathogens. The unspecific protective effects of vaccines were described as soon as the smallpox vaccination was introduced in the 19th century, as physicians related how people treated with the attenuated Vaccinia virus were also protected against other diseases. ${ }^{67}$ This protective effects are generally related with live attenuated vaccines, such as BCG and measles, and possible smallpox. The mecanisms for these nonspecific effects are still a matter of discussion, but its beneficial effects have been extensively utilized, being BCG the most explored of all. The protective effects of BCG vaccination have been proved in murine models of nonmycobacterial infections ${ }^{68}$ and different studies have shown that BGG vaccination at birth is able to reduce mortality in children by means of reducing lower respiratory tract infections and neonatal sepsis. ${ }^{69}$ Intravesical BCG is the first option for the treatment of bladder cancer and other studies have associated BCG with protection against leishmaniasis or asthma. ${ }^{70,71}$

With regard to these nonspecific effects, there is a growing body of evidence that BCG is one of the most powerful agents capable of the induction of trained immunity in human monocytes, causing an strong enhancement of glycolysis, glutamine metabolism, and histone methylation marks. ${ }^{48}$ The relevance of these mechanisms was further validated by a recently published study that showed that BCG vaccination of human volunteers reduced yellow fever viremia through the induction of epigenetic reprogramming of their monocytes and the production of IL- $1 \beta$ that acts as a key mediator of the development of trained immunity. ${ }^{72}$ Kleinnijenhuis et al. showed that BCG vaccination of healthy volunteers led to the production of significantly higher levels of IFN- $\gamma$ and enhanced release of monocyte-derived cytokines, such as TNF and IL-1 $\beta$, in response to unrelated bacterial and fungal pathogens. These immunostimulatory effects were maintained up to 3 months after the initial vaccination. ${ }^{73}$ Higgins et al. reviewed the results from 34 birth cohorts and analyzed the association of BCG, DTP, and measles-containing vaccines (MCV) with childhood mortality concluding that the administration of BCG and MCV reduced overall mortality by more than what would be expected just through their direct effects on the diseases they prevent. ${ }^{74}$ Berendsen et al. found a time-dependent pattern of nonspecific effects of vaccination with
BCG, DTP, and measles, and stunting, with positive associations for vaccinations given early in human life and negative associations for vaccinations given later in infancy. ${ }^{75}$

\section{2 | Sepsis}

According to recent reports, sepsis affects between 20 and 30 million people worldwide every year, remaining a leading cause of death. ${ }^{76}$ The induction of hyporesponsiveness, or tolerance, acts a defense mechanism that decreases the response to inflammation maintaining homeostasis of the host. However, in many cases the immune system is unable to recover from this self-induced suppression, falling into an immunoparalysis state in which the host is unable to stop the progression of the infectious disease. ${ }^{24}$ In the last years, a growing number of studies have shown how long-term reprogramming of cells after exposure to pathogens are of great importance for early cytokine production and for the induction of immunoparalysis and the establishment of disease tolerance. ${ }^{15}$ Monocytes from patients suffering from sepsis-induced immunoparalysis show reduced proinflammatory cytokine production with a defective capacity to mount glycolysis and $\beta$-oxidation. ${ }^{2}$ High levels of lactate and a low clearance rate can help predicting poor outcomes of septic states. ${ }^{77}$

However, the line between the development of tolerance or the induction of training is very thin. The development of immunoparalysis or the induction of trained immunity involves opposing regulation of common pathways ${ }^{20}$ and the blockade of a single step in oxidative phosphorylation switches the potential of LPS-stimulated macrophages from proinflammatory to anti-inflammatory. ${ }^{34}$ Tolerance and training are manifestations of the similar reprogramming processes, but with opposite consequences. Immune status in patients with sepsis or noninfectious systemic inflammatory response síndrome is altered. ${ }^{78}$ It has been known for long that IFN- $\gamma$ and GM-CSF can restore the responsiveness of monocytes from septic patients. ${ }^{79,80}$ Cheng et al. demonstrated that the immunostimulant effects of IFN$\gamma$ in these patients partially relied in the restoration of the metabolic defects of innate immunotolerance by promoting glycolysis. ${ }^{2}$ Administration of $\beta$-glucan to mice induced proliferation of the progenitors of the myeloid lineage, which was associated with enhanced signaling by IL-1 $\beta$ and GM-CSF, and with adaptations in glucose metabolism and cholesterol biosynthesis, improving responses to secondary LPS challenge and protecing from myeloid-induced immunosuppression in mice. ${ }^{28}$ The intravenous injection of bone marrow stromal cells improved survival and organ function after cecal ligation and puncture in mice. ${ }^{81}$

\section{3 | Cancer}

The immune system exerts potent antitumor effects when it is efficiently activated. However, besides fighting cancer, the immune system seems to be involved in the development and progression of many cancer types. A unifying feature of cancer is long-term inflammation. The tumor environment fuctionally reprograms the immune cells to counteract the antitumor effects and promote inflammation, which is often associated with a progression of the disease. ${ }^{82}$ Since innate cells infiltrate the area around a tumor, they become part of a complex 
tumor microenvironment. The presence of inflammatory cells in the tumor microenvironment often promotes tumor progression instead of destruction, as they can promote tissue remodeling, angiogenesis, and fibrosis, which are beneficial for tumor growth and increase resistance to pharmacological treatments. ${ }^{83}$ Much of the work done in immunotherapy during the last years has addressed the ways to revert this reprogramming of cells and recover the antitumor properties. Cancer cells promote the differentiation of monocytes into antiinflammatory macrophages, characterized by the production of high amounts of anti-inflammatory cytokines and proangiogenic factors, impairing immune response and promoting tumor growth. ${ }^{84,85}$

There is strong evidence that some types of infections and vaccinations might be related with a protective response against solid tumors. ${ }^{86}$ In this regard, the greatest success of treatment of cancer with stimuli that can induce reprogramming at the innate immunity level, is the utilization of intravesical BCG as the first option for the treatment of superficial, nonmuscle-invasive bladder cancer. ${ }^{87}$ The antitumor effects of BCG is known since 1929, when a report of necropsies from tuberculosis patients described that the incidence of neoplastic malignancies in tuberculosis patients was significantly lower than in the control group. ${ }^{88}$ The mechanism of action of BCG in this therapy is still a matter of controversy, but relies on a complex immune response which involves the recruitment, activation, and production of proinflammatory cytokines by monocytes, neutrophils, NK cells, and T lymphocytes. ${ }^{89,90}$ An efficient long-term cell reprogramming is necessary to warrant the efficacy of the treatments, as shown by the failure to develop durable responses after treatment with checkpoint inhibitors in cancer patients due to epigenetic stability of exhausted T cells. ${ }^{91,92}$ The transcription factor HIF1 $\alpha$, which plays a central role in the metabolic switch to glycolysis in activated monocytes, has also been implicated in the regulation of many of the genes responsable for metabolic reprogramming in cancer cells. ${ }^{93}$ Mutations affecting SDH complex subunits, that lead to the accummulation of succinate have been identified in familiar paragangliomas. ${ }^{94}$

\section{4 | Inflammatory diseases}

The incidence of chronic and degenerative diseases with an inflammatory or auto-immune basis has augmented over the last years, especially in societies with higher socio-economic development. These diseases have flourished in the last decades as an epidemic outbreak and its incidence has increased together with the degree of industrialization. ${ }^{95,96}$ These diseases are mainly metabolic, cardiovascular, digestive, neurologic, and allergic. ${ }^{97,98}$ Chronic inflammation is the common feature of these diseases. Several studies have demonstrated that reprogramming of the innate immune system represents one of the central features involved in the outbreak and upkeep of chronic inflammation. ${ }^{14,26}$

Progression of atherosclerosis has been mechanistically linked with activation and recruitment of monocyte-derived macrophages. ${ }^{99}$ Accummulation of cholesterol crystals triggers a number of inflammatory pathways that drive cardiovascular diseases. There is evidence that the activation of the cholesterol pathway through the action of the intermediate compound mevalonate, triggers epigenetic reprogramming and is essential for the induction of trained immunity. ${ }^{14}$ In this regard, hyper immunoglobulin D syndrome patients, who accummulate mevalonate, have a constitutive trained imunity phenotype with higher cytokine production. ${ }^{14}$ Western diet influences myelopoiesis and induces transcriptional and functional reprogramming of myeloid precursors, leading to long-term systemic inflammation and enhanced responses to LPS that persisted after shifting back to chow diet, by a mechanism dependent of NLRP3 and the IL-1 pathway. ${ }^{26}$ The influence of diet also influences the activity of the sirtuin family of deacetylase enzymes. The levels of expression of NAD+ and sirtuin enzymes can adapt to metabolic state. SIRT1-mediated acetylation of PGC1 $\alpha$ activates transcription of genes important to adaptation to starvation. ${ }^{100}$ In obesity, macrophages accummulate in the adipose tissue and drive chronic inflammation, promoting insulin resistance. ${ }^{101}$ Metabolic activation, including fatty acid oxidation, glycolysis, and glutaminolysis in obese individuals was associated with diabetes outcome. ${ }^{102}$ Hypoglycemia has been linked to increased leukocyte counts and cytokine levels, leading to chronic systemic inflammation. ${ }^{103,104}$

Microbiota plays a central role in the development and function of the gut innate immune system, as germ-free and gnotobiotic animals show large defect in the development and maturation of Peyer's patches, mesenteric lymph nodes, and lymphoid follicles. ${ }^{105}$ Perturbations of gut microbiota affect the interplay between microbiota and host cells resulting in an increased pathogenesis of inflammatory diseases, such as inflammatory bowel disease, ${ }^{106}$ in which pathogenic intestinal bacteria perpetuate inflammation of the gut through disruption of tolerance. In Crohn's disease, the interactions between the bacterial flora and the mucosal immune system are facilitated by a decreased bacterial clearance by macrophages with a defective secretion of proinflammatory cytokines. ${ }^{107}$ The interaction between the immune system and microbiota induces long-term reprogramming at local and systemic levels, inducing suppression of inflammatory responses to food and orally ingested antigens in order to limit tissue inflammation and microbial translocation. ${ }^{108,109}$ Gut bacteria regulate the activity of intestinal phagocytes, such as macrophages and neutrophils, through continuous priming of resident macrophages to release large amounts of IL-10, what promotes the induction of tolerance to intestinal bacteria and prevents the development os systemic inflammation. ${ }^{110,111}$ Microbiota has also been shown to trigger systemic training by inducing long-term reprogramming of bone marrow dendritic cells provoding IL-17A-dependent protection against protozoal colitis in a mouse model of this disease. ${ }^{112}$ Of note, stromal cells of the intestine of inflammatory bowel disease patients also exhibit stable functional long-term alterations as a result of chronic exposure to an inflammatory environment, ${ }^{113,114}$ which are likely to be mediated by epigenetic and/or metabolic reprogramming. ${ }^{115}$

\section{6 | CONCLUDING REMARKS}

Preservation of homeostasis is achieved not only through containment or erradication of pathogens, but also through adaptations that prepare the host for a secondary challenge. In this regard, innate immune 


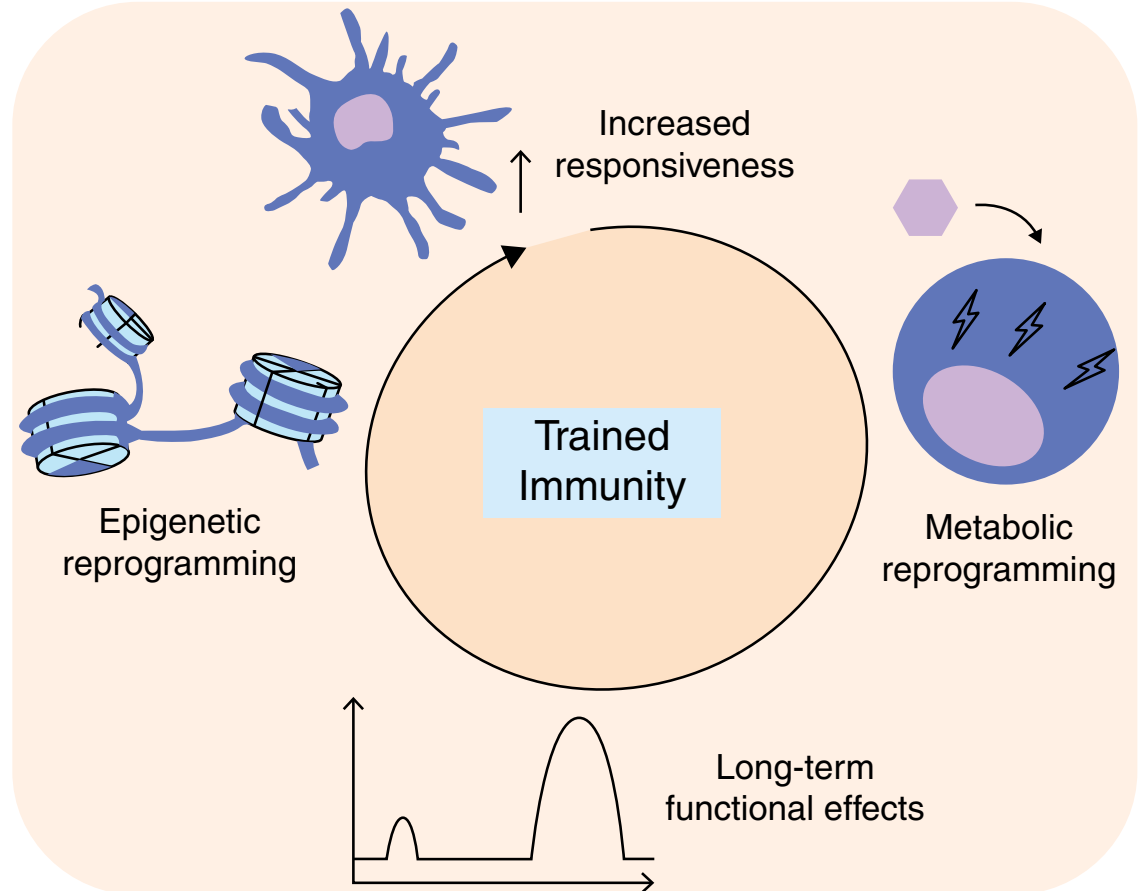

FIGURE 4 Overview of the hallmarks of trained immunity. Trained immunity involves deep changes in metabolic pathways and in the epigenetic landscape of the cell, inducing longterm reprogramming and increasing the capacity of the innate immune cells to respond to secondary stimulation training causes long-term alterations in mature innate immune cells, enabling a robust innate host response to a secondary stimulus (Fig. 4). The evidence reviewed here demonstrates that long-term reprogramming of the innate immune system (trained immunity) is a fundamental property for host defense, being a fundamental complement for the antigen-specific immunological memory mediated by lymphocytes. Trained immunity mediates an increase in the responsiveness of the cells, as shown by the amplification of both pro- and anti-inflammatory cytokine production, ROS production, and metabolic activation. ${ }^{29}$ Further research is required to elucidate the duration of innate immune memory and its effect on the innate immune cell precursors in the bone marrow and tissue macrophage populations. The progress of proteomics, metabolomics, transcriptomics, and epigenomics will permit the identification of the potential novel features of trained immunity and how the different genetic hallmarks of the individuals affect the quality and the range of the reprogramming.

The modulation of cellular reprogramming is also believed to have great potential as a therapeutic tool in immune-mediated diseases. By elucidating the potential role of trained immunity in these diseases, new steps can be made in better understanding their pathophysiology and potentially lead to new clinical approaches. In inflammatory diseases in which monocytes and macrophages are skewed toward a proinflammatory profile, inhibition of certain metabolic or epigenetic pathways involved in cellular reprogramming may represent a valid approach to target. Induction of metabolic pathways important for trained immunity can be used be attempted to restore immune function during sepsis-induced immune paralysis. Inducers of cellular reprogramming such as $\beta$-glucan or muramyl dipeptide have shown potential as a treatment or adjuvant for osteosarcoma, ${ }^{116}$ influenza, ${ }^{117}$ or skin lesions, ${ }^{118}$ among others. The actions of innate immune memory in precursor cells of the innate immune system in the bone marrow can be therapeutically exploited to counteract the adverse effects of chemotherapy induced myelosuppression. ${ }^{28}$ Cellular metabolic pathways for the targeting of reprogramming may also represent a novel therapeutic approach in immunodeficiencies as well as for the modulation of the potentially deleterious consequences of trained immunity in autoinflammatory diseases. All these observations provide new insights for the development of novel therapeutic strategies to reverse the pathological conditons associated with the long-term reprogramming of the innate immune system.

\section{ACKNOWLEDGMENTS}

M.G.N. is supported by an ERC Consolidator grant (\#310372) and by a Spinoza Grant of the Netherlands Organization for Scientific Research.

\section{AUTHORSHIP}

J.D.-A. wrote the first draft and M.N. made revisions.

\section{DISCLOSURE}

The authors declare no competing interests.

\section{REFERENCES}

1. McHeyzer-Williams LJ, McHeyzer-Williams MG. Antigen-specific memory B cell development. Annu Rev Immunol. 2005;23:487-513.

2. Cheng S-C, Scicluna BP, Arts RJW, et al. Broad defects in the energy metabolism of leukocytes underlie immunoparalysis in sepsis. Nat Immunol. 2016;17:406-413.

3. O'neill LAJ. Glycolytic reprogramming by TLRs in dendritic cells. Nat Immunol. 2014;15:314-315.

4. Hellwig D, Voigt J, Bouzani M, et al. Candida albicans induces metabolic reprogramming in human NK cells and responds to perforin with a zinc depletion response. Front Microbiol. 2016;7:750.

5. Kachroo A, Robin GP. Systemic signaling during plant defense. Curr Opin Plant Biol. 2013;16:527-533. 
6. Quintin J, Saeed S, Martens JHA, et al. Candida albicans infection affords protection against reinfection via functional reprogramming of monocytes. Cell Host Microbe. 2012;12:223-232.

7. Netea MG, Quintin J, Van Der Meer JWM. Trained immunity: a memory for innate host defense. Cell Host Microbe. 2011;9: 355-361.

8. Jones DD, Wilmore JR, Allman D. Cellular dynamics of memory B cell populations: IgM+ and IgG+ memory B cells persist indefinitely as quiescent cells. J Immunol. 2015;195:4753-4759.

9. Tough DF, Sprent J. Life span of naive and memory t cells. Stem Cells. 1995;13:242-249.

10. Saeed S, Quintin J, Kerstens HHD, et al. Epigenetic programming of monocyte-to-macrophage differentiation and trained innate immunity. Science. 2014;345:1251086-1251086.

11. Cheng S-C, Quintin J, Cramer R, et al. mTOR- and HIF-1 -mediated aerobic glycolysis as metabolic basis for trained immunity. Science. 2014;345:1250684-1250684.

12. Arts RJW, Novakovic B, ter Horst R, et al. Glutaminolysis and fumarate accumulation integrate immunometabolic and epigenetic programs in trained immunity. Cell Metab. 2016;24:807-819.

13. Kleinnijenhuis J, Quintin J, Preijers F, et al. Long-lasting effects of BCG vaccination on both heterologous Th1/Th17 responses and innate trained immunity. J Innate Immun. 2014;6:152-158.

14. Bekkering S, Arts RJW, Novakovic B, et al. Metabolic induction of trained immunity through the mevalonate pathway. Cell. 2018;172:135-146.e9.

15. van der Poll T, van de Veerdonk FL, Scicluna BP, Netea MG. The immunopathology of sepsis and potential therapeutic targets. Nat Rev Immunol. 2017;17:407-420.

16. Stienstra R, Netea-Maier RT, Riksen NP, Joosten LAB, Netea MG. Specific and complex reprogramming of cellular metabolism in myeloid cells during innate immune responses. Cell Metab. 2017;26:142-156.

17. Janeway CA, Medzhitov R. Innate immune recognition. Annu Rev Immunol. 2002;20:197-216.

18. Bowdish DME, Loffredo MS, Mukhopadhyay S, Mantovani A, Gordon S. Macrophage receptors implicated in the "adaptive" form of innate immunity. Microbes Infect. 2007;9:1680-1687.

19. Chen GY, Nuñez G. Sterile inflammation: sensing and reacting to damage. Nat Rev Immunol. 2010;10:826-837.

20. Novakovic B, Habibi E, Wang S-Y, et al. $\beta$-Glucan reverses the epigenetic state of LPS-induced immunological tolerance. Cell. 2016;167:1354-1368.e14.

21. van de Veerdonk FL, Marijnissen RJ, Kullberg BJ, et al. The macrophage mannose receptor induces IL-17 in response to Candida albicans. Cell Host Microbe. 2009;5:329-340.

22. Weis S, Carlos AR, Moita MR, et al. Metabolic adaptation establishes disease tolerance to sepsis. Cell. 2017;169:1263-1275.e14.

23. Carré JE, Orban J-C, Re L, et al. Survival in critical illness is associated with early activation of mitochondrial biogenesis. Am J Respir Crit Care Med. 2010;182:745-751.

24. Medzhitov R, Schneider DS, Soares MP. Disease tolerance as a defense strategy. Science. 2012;335:936-941.

25. Patel AA, Zhang Y, Fullerton JN, et al. The fate and lifespan of human monocyte subsets in steady state and systemic inflammation. J Exp Med. 2017;214:1913-1923.

26. Christ A, Günther P, Lauterbach MAR, et al. Western diet triggers NLRP3-dependent innate immune reprogramming. Cell. 2018;172:162-175.e14.
27. Kaufmann E, Sanz J, Dunn JL, et al. BCG educates hematopoietic stem cells to generate protective innate immunity against tuberculosis. Cell. 2018;172:176-190.e19.

28. Mitroulis I, Ruppova K, Wang B, et al. Modulation of myelopoiesis progenitors is an integral component of trained immunity. Cell. 2018;172:147-161.e12.

29. Bekkering S, Blok BA, Joosten LAB, Riksen NP, van Crevel R, Netea MG. In vitro experimental model of trained innate immunity in human primary monocytes. Clin Vaccine Immunol. 2016;23:926-933.

30. Pearce E, Pearce E. Metabolic pathways in immune cell activation and quiescence. Immunity. 2013;38:633-643.

31. Buck MD, Sowell RT, Kaech SM, Pearce EL, Metabolic Instruction of Immunity. Cell. 2017;169:570-586.

32. Newsholme P, Gordon S, Newsholme EA. Rates of utilization and fates of glucose, glutamine, pyruvate, fatty acids and ketone bodies by mouse macrophages. Biochem J. 1987;242:631-636.

33. Lachmandas E, Boutens L, Ratter JM, et al. Microbial stimulation of different Toll-like receptor signalling pathways induces diverse metabolic programmes in human monocytes. Nat Microbiol. 2016;2:16246.

34. Mills EL, Kelly B, Logan A, et al. Succinate dehydrogenase supports metabolic repurposing of mitochondria to drive inflammatory macrophages. Cell. 2016;167:457-470.e13.

35. Mills EL, Ryan DG, Prag HA, et al. Itaconate is an anti-inflammatory metabolite that activates Nrf2 via alkylation of KEAP1. Nature. 2018.

36. Bambouskova M, Gorvel L, Lampropoulou V, et al. Electrophilic properties of itaconate and derivatives regulate the $\mathrm{I}_{\kappa} \mathrm{B} \zeta$-ATF3 inflammatory axis. Nature. 2018;1.

37. Domínguez-Andrés J, Feo-Lucas L, Minguito de la Escalera M, González L, López-Bravo M, Ardavín C. Inflammatory Ly6C high monocytes protect against candidiasis through IL-15-Driven NK cell/neutrophil activation. Immunity. 2017;46:1059-1072.e4.

38. van Raam BJ, Sluiter W, de Wit E, Roos D, Verhoeven AJ, Kuijpers TW. Mitochondrial membrane potential in human neutrophils is maintained by complex III activity in the absence of supercomplex organisation. PLoS One. 2008;3:e2013.

39. Rodríguez-Espinosa O, Rojas-Espinosa O, Moreno-Altamirano MMB, López-Villegas EO, Sánchez-García FJ. Metabolic requirements for neutrophil extracellular traps formation. Immunology. 2015;145:213-224

40. Zaccagnino P, Saltarella M, Maiorano S, et al. An active mitochondrial biogenesis occurs during dendritic cell differentiation. Int J Biochem Cell Biol. 2012;44:1962-1969.

41. Le Naour F, Hohenkirk L, Grollea A, et al. Profiling changes in gene expression during differentiation and maturation of monocytederived dendritic cells using both oligonucleotide microarrays and proteomics. J Biol Chem. 2001;276:17920-17931.

42. Krawczyk CM, Holowka T, Sun J, et al. Toll-like receptor-induced changes in glycolytic metabolism regulate dendritic cell activation. Blood. 2010;115:4742-4749.

43. Malinarich F, Duan K, Hamid RA, et al. High mitochondrial respiration and glycolytic capacity represent a metabolic phenotype of human tolerogenic dendritic cells. J Immunol. 2015;194: 5174-5186.

44. Keppel MP, Saucier N, Mah AY, Vogel TP, Cooper MA. Activationspecific metabolic requirements for $\mathrm{NK}$ cell IFN- $\gamma$ production. J Immunol. 2015;194:1954-1962.

45. Marçais A, Cherfils-Vicini J, Viant C, et al. The metabolic checkpoint kinase mTOR is essential for IL-15 signaling during the development and activation of NK cells. Nat Immunol. 2014;15:749-757. 
46. Keating SE, Zaiatz-Bittencourt V, Loftus RM, et al. Metabolic reprogramming supports IFN- $\gamma$ production by CD56bright NK cells. J Immunol. 2016;196:2552-2560.

47. Assmann N, O'Brien KL, Donnelly RP, et al. Srebp-controlled glucose metabolism is essential for NK cell functional responses. Nat Immunol. 2017;18:1197-1206.

48. Arts RJW, Carvalho A, La Rocca C, et al. Immunometabolic pathways in BCG-induced trained immunity. Cell Rep. 2016;17:2562-2571.

49. Rui L. Energy metabolism in the liver. Compr Physiol. 2014;4:177-197.

50. O'Sullivan TE, Sun JC, Lanier LL. Natural killer cell memory. Immunity. 2015;43:634-645.

51. Liu TF, Vachharajani VT, Yoza BK, McCall CE. NAD+-dependent Sirtuin 1 and 6 proteins coordinate a switch from glucose to fatty acid oxidation during the acute inflammatory response. J Biol Chem. 2012;287:25758-25769.

52. Le A, Lane AN, Hamaker M, et al. Glucose-independent glutamine metabolism via TCA cycling for proliferation and survival in B cells. Cell Metab. 2012;15:110-121.

53. Jayaram $\mathrm{H}$, Hoelper $\mathrm{D}$, Jain SU, et al. S-adenosyl methionine is necessary for inhibition of the methyltransferase G9a by the lysine 9 to methionine mutation on histone H3. Proc Natl Acad Sci USA. 2016;113:6182-6187.

54. Xu W, Yang H, Liu Y, et al. Oncometabolite 2-hydroxyglutarate is a competitive inhibitor of $\alpha$-ketoglutarate-dependent dioxygenases. Cancer Cell. 2011;19:17-30.

55. Trapp J, Jung M. The role of NAD+ dependent histone deacetylases (sirtuins) in ageing. Curr Drug Targets. 2006;7:1553-1560.

56. Friedmann DR, Marmorstein R. Structure and mechanism of nonhistone protein acetyltransferase enzymes. FEBS J. 2013;280: 5570-5581.

57. Lee KK, Workman JL. Histone acetyltransferase complexes: one size doesn't fit all. Nat Rev Mol Cell Biol. 2007;8:284-295.

58. Shimazu T, Hirschey MD, Newman J, et al. Suppression of oxidative stress by $\beta$-hydroxybutyrate, an endogenous histone deacetylase inhibitor. Science. 2013;339:211-214.

59. Sowter HM, Raval RR, Moore JW, Ratcliffe PJ, Harris AL. Predominant role of hypoxia-inducible transcription factor (Hif)-1alpha versus Hif-2alpha in regulation of the transcriptional response to hypoxia. Cancer Res. 2003;63:6130-6134.

60. Cervera AM, Bayley J-P, Devilee P, McCreath KJ. Inhibition of succinate dehydrogenase dysregulates histone modification in mammalian cells. Mol Cancer. 2009;8:89.

61. Chowdhury R, Yeoh KK, Tian Y-M, et al. The oncometabolite 2hydroxyglutarate inhibits histone lysine demethylases. EMBO Rep. 2011;12:463-469.

62. Figueroa ME, Lugthart S, LiY, et al. DNA methylation signatures identify biologically distinct subtypes in acute myeloid leukemia. Cancer Cell. 2010;17:13-27.

63. Koivunen $\mathrm{P}$, Lee $\mathrm{S}$, Duncan CG, et al. Transformation by the (R)enantiomer of 2-hydroxyglutarate linked to EGLN activation. Nature. 2012;483:484-488.

64. Turcan S, Rohle D, Goenka A, et al. IDH1 mutation is sufficient to establish the glioma hypermethylator phenotype. Nature. 2012;483:479-483.

65. Foster SL, Hargreaves DC, Medzhitov R. Gene-specific control of inflammation by TLR-induced chromatin modifications. Nature. 2007;447:972-978.

66. Ostuni R, Piccolo V, Barozzi I, et al. Latent enhancers activated by stimulation in differentiated cells. Cell. 2013;152:157-171.
67. Mayr A. Taking advantage of the positive side-effects of smallpox vaccination. J Vet Med Ser B. 2004;51:199-201.

68. Blok BA, Arts RJW, van Crevel R, Benn CS, Netea MG. Trained innate immunity as underlying mechanism for the long-term, nonspecific effects of vaccines. J Leukoc Biol. 2015;98:347-356.

69. Aaby $\mathrm{P}$, Roth $\mathrm{A}$, Ravn $\mathrm{H}$, et al. Randomized trial of BCG vaccination at birth to low-birth-weight children: beneficial nonspecific effects in the neonatal period? J Infect Dis. 2011;204:245-252.

70. Fortier AH, Mock BA, Meltzer MS, Nacy CA. Mycobacterium bovis BCG-induced protection against cutaneous and systemic Leishmania major infections of mice. Infect Immun. 1987;55: 1707-1714.

71. El-Zein M, Parent ME, Benedetti A, Rousseau MC. Does BCG vaccination protect against the development of childhood asthma? A systematic review and meta-analysis of epidemiological studies. Int J Epidemiol. 2010;39:469-486.

72. Arts RJW, Moorlag SJCFM, Novakovic B, et al. BCG vaccination protects against experimental viral infection in humans through the induction of cytokines associated with trained immunity. Cell Host Microbe. 2018;23:89-100.e5.

73. Kleinnijenhuis J, Quintin J, Preijers F, et al. Bacille Calmette-Guerin induces NOD2-dependent nonspecific protection from reinfection via epigenetic reprogramming of monocytes. Proc Natl Acad Sci USA. 2012;109:17537-17542

74. Higgins JPT, Soares-Weiser K, López-López JA, et al. Association of BCG, DTP, and measles containing vaccines with childhood mortality: systematic review. BMJ. 2016;355:i5170.

75. Berendsen MLT, Smits J, Netea MG, van der Ven A. Nonspecific effects of vaccines and stunting: timing may be essential. EBioMedicine. 2016:8:341-348.

76. Martin GS. Sepsis, severe sepsis and septic shock: changes in incidence, pathogens and outcomes. Expert Rev Anti Infect Ther. 2012;10:701-706.

77. Garcia-Alvarez M, Marik P, Bellomo R. Sepsis-associated hyperlactatemia. Crit Care. 2014;18:503.

78. Cavaillon J-M, Adrie C, Fitting C, Adib-Conquy M. Reprogramming of circulatory cells in sepsis and SIRS. J Endotoxin Res. 2005;11: 311-320.

79. Döcke WD, Randow F, Syrbe U, et al. Monocyte deactivation in septic patients: restoration by IFN-gamma treatment. Nat Med. 1997;3:678-681.

80. Flohé S, Lendemans S, Selbach C, et al. Effect of granulocytemacrophage colony-stimulating factor on the immune response of circulating monocytes after severe trauma. Crit Care Med. 2003;31: 2462-2469

81. Németh K, Leelahavanichkul A, Yuen PST, et al. Bone marrow stromal cells attenuate sepsis via prostaglandin E2-dependent reprogramming of host macrophages to increase their interleukin-10 production. Nat Med. 2009;15:42-49.

82. Shalapour S, Karin M. Immunity, inflammation, and cancer: an eternal fight between good and evil. J Clin Invest. 2015;125:3347-3355.

83. Quail DF, Joyce JA. Microenvironmental regulation of tumor progression and metastasis. Nat Med. 2013;19:1423-1437.

84. Mantovani A, Sozzani S, Locati M, Allavena P, Sica A. Macrophage polarization: tumor-associated macrophages as a paradigm for polarized M2 mononuclear phagocytes. Trends Immunol. 2002;23: 549-555.

85. Heusinkveld M, van der Burg SH. Identification and manipulation of tumor associated macrophages in human cancers. J Transl Med. 2011;9:216. 
86. Krone B, Kölmel KF, Grange JM. The biography of the immune system and the control of cancer: from St Peregrine to contemporary vaccination strategies. BMC Cancer. 2014;14:595.

87. Kiselyov A, Bunimovich-Mendrazitsky S, Startsev V. Treatment of non-muscle invasive bladder cancer with Bacillus CalmetteGuerin (BCG): biological markers and simulation studies. BBA Clin. 2015;4:27-34.

88. Pearl R. Cancer and tuberculosis. Am J Epidemiol. 1929;9: 97-159.

89. Redelman-Sidi G, Glickman MS, Bochner BH. The mechanism of action of BCG therapy for bladder cancer-a current perspective. Nat Rev Urol. 2014;11:153-162.

90. Luo Y, Knudson MJ. Mycobacterium bovis bacillus Calmette-Guérininduced macrophage cytotoxicity against bladder cancer cells. Clin Dev Immunol. 2010;2010:357591.

91. Pauken KE, Sammons MA, Odorizzi PM, et al. Epigenetic stability of exhausted T cells limits durability of reinvigoration by PD-1 blockade. Science. 2016;354:1160-1165.

92. Sen DR, Kaminski J, Barnitz RA, et al. The epigenetic landscape of $T$ cell exhaustion. Science. 2016;354:1165-1169.

93. Denko NC. Hypoxia, HIF1 and glucose metabolism in the solid tumour. Nat Rev Cancer. 2008;8:705-713.

94. Rutter J, Winge DR, Schiffman JD. Succinate dehydrogenase Assembly, regulation and role in human disease. Mitochondrion. 2010;10:393-401

95. Rose DP, Gracheck PJ, Vona-Davis L. The interactions of obesity, inflammation and insulin resistance in breast cancer. Cancers (Basel). 2015;7:2147-2168.

96. Manzel A, Muller DN, Hafler DA, Erdman SE, Linker RA, Kleinewietfeld M. Role of "Western diet" in inflammatory autoimmune diseases. Curr Allergy Asthma Rep. 2014;14:404.

97. Kim B-J, Lee S-Y, Kim H-B, Lee E, Hong S-J. Environmental changes, microbiota, and allergic diseases. Allergy Asthma Immunol Res. 2014;6:389-400.

98. Uluçkan Ö, Wagner EF. Chronic systemic inflammation originating from epithelial tissues. FEBS J. 2017;284:505-516.

99. Ley K, Miller YI, Hedrick CC. Monocyte and macrophage dynamics during atherogenesis. Arterioscler Thromb Vasc Biol. 2011;31: 1506-1516.

100. Hirschey MD, Shimazu T, Jing E, et al. SIRT3 deficiency and mitochondrial protein hyperacetylation accelerate the development of the metabolic syndrome. Mol Cell. 2011:44:177-190.

101. Chung K-J, Chatzigeorgiou A, Economopoulou M, et al. A selfsustained loop of inflammation-driven inhibition of beige adipogenesis in obesity. Nat Immunol. 2017;18:654-664.

102. Boutens L, Hooiveld GJ, Dhingra S, Cramer RA, Netea MG, Stienstra R. Unique metabolic activation of adipose tissue macrophages in obesity promotes inflammatory responses. Diabetologia. 2018.

103. Razavi Nematollahi L, Kitabchi AE, Stentz FB, et al. Proinflammatory cytokines in response to insulin-induced hypoglycemic stress in healthy subjects. Metabolism. 2009;58:443-448.

104. Ceriello A, Gallo M, Candido R, et al. Personalized therapy algorithms for type 2 diabetes: a phenotype-based approach. Pharmgenomics Pers Med. 2014:7:129-136.
105. Bouskra D, Brézillon C, Bérard M, et al. Lymphoid tissue genesis induced by commensals through NOD1 regulates intestinal homeostasis. Nature. 2008;456:507-510.

106. Kim D, Zeng MY, Núñez G. The interplay between host immune cells and gut microbiota in chronic inflammatory diseases. Exp Mol Med. 2017:49:e339.

107. Smith AM, Rahman FZ, Hayee B, et al. Disordered macrophage cytokine secretion underlies impaired acute inflammation and bacterial clearance in Crohn's disease. J Exp Med. 2009;206:1883-1897.

108. Chistiakov DA, Bobryshev Y V, Kozarov E, Sobenin IA, Orekhov AN. Intestinal mucosal tolerance and impact of gut microbiota to mucosal tolerance. Front Microbiol. 2015;5:781.

109. Luu M, Steinhoff U, Visekruna A. Functional heterogeneity of gutresident regulatory T cells. Clin Transl Immunol. 2017;6:e156.

110. Kamada N, Núñez G. Regulation of the immune system by the resident intestinal bacteria. Gastroenterology. 2014;146:1477-1488.

111. Rivollier A, He J, Kole A, Valatas V, Kelsall BL. Inflammation switches the differentiation program of Ly6Chi monocytes from antiinflammatory macrophages to inflammatory dendritic cells in the colon. $J$ Exp Med. 2012;209:139-155.

112. Burgess SL, Buonomo E, Carey $\mathrm{M}$, et al. Bone marrow dendritic cells from mice with an altered microbiota provide interleukin 17adependent protection against Entamoeba histolytica colitis. MBio. 2014;5:e01817-14.

113. McKaig BC, Hughes $K$, Tighe PJ, Mahida YR. Differential expression of TGF- $\beta$ isoforms by normal and inflammatory bowel disease intestinal myofibroblasts. Am J Physiol Physiol. 2002;282:C172-C182.

114. McKaig BC, McWilliams D, Watson SA, Mahida YR. Expression and regulation of tissue inhibitor of metalloproteinase- 1 and matrix metalloproteinases by intestinal myofibroblasts in inflammatory bowel disease. Am J Pathol. 2003;162:1355-1360.

115. Owens BMJ. Inflammation, innate immunity, and the intestinal stromal cell niche: opportunities and challenges. Front Immunol. 2015;6:319.

116. Meyers PA, Schwartz CL, Krailo MD, et al. Osteosarcoma: the addition of muramyl tripeptide to chemotherapy improves overall survival-a report from the Children's Oncology Group. J Clin Oncol. 2008;26:633-638.

117. Muramatsu D, Iwai A, Aoki S, et al. $\beta$-glucan derived from aureobasidium pullulans is effective for the prevention of influenza in mice. PLoS One. 2012;7:e41399.

118. Sugiyama $A$, Hata $S$, Suzuki $K$, et al. Oral administration of paramylon, a $\beta$-1,3-D-Glucan isolated from euglena gracilis $Z$ inhibits development of atopic dermatitis-like skin lesions in NC/Nga mice. $J$ Vet Med Sci. 2010;72:755-763.

119. Domínguez-Andrés J, Arts RJW, ter Horst R, et al. Rewiring monocyte glucose metabolism via C-type lectin signaling protects against disseminated candidiasis. PLOS Pathog. 2017;13:e1006632.

How to cite this article: Dominguez-Andres J, Netea MG. Long-term reprogramming of the innate immune system. J Leukoc Biol. 2019;105:329-338. https://doi.org/10.1002/JLB. MR0318-104R 